\title{
Induced transitions between Wannier ladders
}

\author{
M. Glück, M. Hankel, A. R. Kolovsky and H. J. Korsch \\ Fachbereich Physik, Universität Kaiserslautern, D-67653 Kaiserslautern, Germany
}

November 25, 1999

\begin{abstract}
We study the transitions between the ground and excited Wannier states induced by a weak ac field. Because the upper Wannier states are several order of magnitude less stable than the ground states, these transitions decrease the global stability of the system characterized by the rate of probability leakage or decay rate. Using nonhermitian resonant perturbation theory we obtain an analytical expression for this induced decay rate. The analytical results are compared with exact numerical calculations of the system decay rate.
\end{abstract}

PACS. : $42.50 . \mathrm{Hz}, 03.65 .-\mathrm{w}, 73.20 . \mathrm{Dx}$

\section{Introduction}

In our preceding papers [1] (see also [2]) we have studied the spectral properties of the Wannier-Stark system

$$
\widehat{H}_{0}=\hat{p}^{2} / 2+\cos x+F x .
$$

In this paper we discuss the transitions in the system (1) induced by the time-periodic perturbation

$$
\widehat{H}=\widehat{H}_{0}+F_{\omega} x \cos (\omega t) .
$$

Here we address this problem in relation to the experiments with cold atoms in an accelerated standing wave [3]. However, the results obtained have a general validity and can be applied, for example, to biased semiconductor superlattices [4] as well.

The "visiting card" of the problem under study is the transition scheme depicted in Fig. 1. This scheme shows the possible transitions between the ground and excited Wannier-Stark states induced by the periodic driving. By scanning the frequency $\omega$ of the driving force one sequentially activates these transitions and the upper levels become populated. Because the upper levels are typically very unstable, the system rapidly decays there, which is reflected in the depletion rate in the experiments with cold atoms or in the response to optical excitation in the experiments with semiconductor superlattices. On the formal level the experimentally measured quantities are directly related to the rate $\boldsymbol{\Gamma}(\omega)$ of probability leakage $P(t)=\exp [-\boldsymbol{\Gamma}(\omega) t]:$

$$
\boldsymbol{\Gamma}(\omega)=\frac{\mathrm{d} \ln P}{\mathrm{~d} t},
$$

which is our main object of study. As follows from the transition scheme, the function (3) should exhibit a number of equidistant peaks with spacing given by the Bloch frequency $\omega_{B}=2 \pi F / \hbar$.
Recently we have suggested an exact numerical method for calculating $\boldsymbol{\Gamma}(\omega)$ [5]. In the present paper we study the function (3) analytically. Although the general structure of $\boldsymbol{\Gamma}(\omega)$ is obvious, little is known about its more specific details such as number of visible lines, their relative intensities, etc. The reason for this was that - to give a reliable prediction - one has to find the metastable WannierStark states, which was a challenging problem until quite recently [6]. In what follows we derive an analytical expression for $\boldsymbol{\Gamma}(\omega)$ and describe a method for calculating the relative intensities and shapes of the lines.

\section{A two-state system}

It is instructive to discuss the dynamics of the transitions in a two-state system constructed from any of the ground $\Psi_{0}^{l}(x)$ and excited $\Psi_{1}^{l^{\prime}}(x)$ Wannier-Stark states (see Fig. 1). When the frequency of the periodic driving is close to the resonant frequency between two Wannier levels the system Hamiltonian can be approximated by a $2 \times 2$ matrix of the form

$$
H_{\text {eff }}^{l, l^{\prime}}=\left(\begin{array}{cc}
\mathcal{E}_{1}^{l^{\prime}} & 0 \\
0 & \mathcal{E}_{0}^{l}
\end{array}\right)+F_{\omega} \cos (\omega t)\left(\begin{array}{ll}
V_{1,1}^{l^{\prime}, l^{\prime}} & V_{1,0}^{l, l^{\prime}} \\
V_{0,1}^{l^{\prime}, l} & V_{0,0}^{l, l}
\end{array}\right),
$$

where $V_{\alpha, \beta}^{l, l^{\prime}}=\left\langle\Psi_{\alpha}^{l}|x| \Psi_{\beta}^{l^{\prime}}\right\rangle$ are the transition matrix elements. In Eq. (4), $\mathcal{E}_{\alpha}^{l}=E_{\alpha}+\hbar \omega_{B} l-i \Gamma_{\alpha} / 2$ and we can set $\Gamma_{0}=0$ because the decay rate of the ground state is negligible in comparison with the decay rate $\Gamma_{1}$ of the upper state. Within this approximation the total probability leakage of the system is given by the equation

$$
\frac{\mathrm{d} P}{\mathrm{~d} t}=-\frac{\Gamma_{1}}{\hbar} P_{1}(t)
$$

where $P_{1}(t)$ is the population of the upper state. 
Using the rotating wave approximation the Hamiltonian (4) takes the form

$$
H_{e f f}^{l, l^{\prime}}=\left(\begin{array}{cc}
\mathcal{E}_{1}^{l^{\prime}} & F_{\omega} V_{1,0}^{l^{\prime}, l} e^{i \omega t} / 2 \\
F_{\omega} V_{0,1}^{l, l^{\prime}} e^{-i \omega t} / 2 & \mathcal{E}_{0}^{l}
\end{array}\right) .
$$

Thus the (complex) quasienergies are

$$
\begin{gathered}
\mathcal{E}_{ \pm}=\frac{\Delta_{0,1}(L)-i \Gamma_{1} / 2}{2} \\
\pm \frac{1}{2} \sqrt{\left(\Delta_{0,1}(L)-i \Gamma_{1} / 2\right)^{2}+F_{\omega}^{2} V_{0,1}^{2}(L)},
\end{gathered}
$$

where $\Delta_{0,1}(L)=E_{1}-E_{0}+\hbar \omega_{B} L-\hbar \omega$ is the detuning and $L=l^{\prime}-l$ is the "distance" between the states. In Eq. (7) we use the notation

$$
V_{0,1}^{2}(L)=V_{0,1}^{l, l^{\prime}} V_{1,0}^{l^{\prime}, l}, \quad L=l-l^{\prime}
$$

and, for the moment, we assume that the matrix elements $V_{0,1}^{l, l^{\prime}}$ and $V_{1,0}^{l^{\prime}, l}$ are real. (The validity of this approximation will be discussed later on in Sec. 4.) Two limiting cases should be distinguished - the case of weak damping $\Gamma_{1} \ll F_{\omega}\left|V_{0,1}(L)\right|$, and the opposite case of strong damping $\Gamma_{1} \gg F_{\omega}\left|V_{0,1}(L)\right|$. In the former case $P_{1}(t)$ shows decaying Rabi oscillations [see Fig. 2(a)]

$$
P_{1}(t)=\exp \left(-\frac{\Gamma_{1}}{2 \hbar} t\right) \sin ^{2}\left(\frac{F_{\omega} V_{0,1}(L)}{2 \hbar} t\right),
$$

for $P_{1}(0)=0$. (For the sake of simplicity Eq. (9) is displayed for zero detuning.) In the latter case we have an "overdamped" Rabi oscillation [see Fig. 2(b)] and asymptotically

$$
P_{1}(t)=\frac{F_{\omega}^{2} V_{0,1}^{2}(L)}{\Delta_{0,1}^{2}(L)+\left(\Gamma_{1} / 2\right)^{2}} P(t), \quad t \gg 1 .
$$

Substituting Eq. (10) into Eq. (5) we obtain

$$
\boldsymbol{\Gamma}(\omega)=\frac{\mathrm{d} \ln P}{\mathrm{~d} t}=\frac{F_{\omega}^{2} V_{0,1}^{2}(L) \Gamma_{1} / \hbar}{\Delta_{0,1}^{2}(L)+\left(\Gamma_{1} / 2\right)^{2}} .
$$

Thus for a near resonant driving frequency $\left(\Delta_{0,1}(L) \approx 0\right)$ the overall decay rate of the system is proportional to the square of the driving force amplitude $F_{\omega}$ and follows a Lorentzian as a function of the detuning [8]. The overdamped regime is always realized for $F_{\omega} \rightarrow 0$ and was actually observed in the experiment [3]. In what follows we restrict ourselves exclusively to this case.

To conclude this section we note that the result (11) can be directly obtained from Eq. (7) as

$$
\boldsymbol{\Gamma}(\omega)=\operatorname{Im}\left(\mathcal{E}_{-}\right),
$$

where $\mathcal{E}_{-}$is the more stable [minus sign in Eq. (7)] quasienergy. Equation (12) has a simple physical interpretation. Indeed, an arbitrary initial state of the system can be represented as a sum over the quasienergy states. However, asymptotically only the most stable quasienergy state survives. Thus for $t \rightarrow \infty$ the total decay rate of the system is given by the imaginary part of this quasienergy.

\section{Transition matrix elements: Theory}

To go further we need information about the transition matrix elements

$$
V_{\alpha, \beta}^{l, l^{\prime}}=\left\langle\Psi_{\alpha}^{l}|x| \Psi_{\beta}^{l^{\prime}}\right\rangle .
$$

In this section we describe the method for calculating them. One should recall from the very beginning that the $\Psi_{\alpha}^{l}(x)$ in Eq. (13) are resonance states and, therefore, we distinguish between the left $\left\langle\Psi_{\alpha}^{l}\right|$ and right $\left|\Psi_{\alpha}^{l}\right\rangle$ eigenfunctions.

To calculate the matrix elements (13) we employ the relation between the Wannier-Stark states $\Psi_{\alpha}^{l}(x)$ and the Wannier-Bloch states $\psi_{\alpha}^{k}(x)[6]$

$$
\Psi_{\alpha}^{l}(x)=\int_{-1 / 2}^{1 / 2} \mathrm{~d} k e^{-i 2 \pi l k} \psi_{\alpha}^{k}(x) .
$$

The latter states are Bloch waves

$$
\psi_{\alpha}^{k}(x)=e^{i k x} \phi_{\alpha}^{k}(x), \quad \phi_{\alpha}^{k}(x)=\frac{1}{2 \pi} \sum_{n} c_{\alpha}^{k}(n) e^{i n x} .
$$

with $k$ being the quasimomentum. Numerically one finds the expansion coefficients $c_{\alpha}^{k}(n)$ in Eq. (15) as the right (left) eigenvectors of some nonunitary matrix $B$ (see Eq. (33) in Ref. [1]).

Using Eq. (14) and Eq. (15) we obtain the intermediate relation

$$
x \Psi_{\alpha}^{l}=2 \pi l \Psi_{\alpha}^{l}+i \int_{-1 / 2}^{1 / 2} \mathrm{~d} k e^{-i 2 \pi l k} e^{i k x} \frac{\mathrm{d}}{\mathrm{d} k} \phi_{\beta}^{k} .
$$

Then substituting Eqs. (14)-(16) into Eq. (13) we have

$$
V_{\alpha, \beta}^{l, l^{\prime}}=2 \pi l \delta_{\alpha, \beta} \delta_{l, l^{\prime}}+i \int_{-1 / 2}^{1 / 2} \mathrm{~d} k e^{i 2 \pi\left(l-l^{\prime}\right) k}\left\langle\phi_{\alpha}^{k} \mid \frac{\mathrm{d}}{\mathrm{d} k} \phi_{\beta}^{k}\right\rangle .
$$

It is shown in Ref. $[1,6]$ that the function $\phi_{\alpha}^{k}(x)$ with an arbitrary value of the quasimomentum $k$ can be generated from $\phi_{\alpha}^{0}(x)$ by the transformation

$$
\left|\phi_{\alpha}^{k}\right\rangle=\widehat{W}(t)\left|\phi_{\alpha}^{0}\right\rangle, \quad\left\langle\phi_{\alpha}^{k}\right|=\left\langle\phi_{\alpha}^{0}\right| \widehat{W}^{-1}(t),
$$

where

$$
\begin{gathered}
\widehat{W}(t)=\exp \left(-i \frac{\mathcal{E}_{\alpha} t}{\hbar}\right) \\
\times \widehat{\exp }\left\{-\frac{i}{\hbar} \int_{0}^{t}\left[\frac{\left(\hat{p}+F t^{\prime}\right)^{2}}{2}+\cos x\right] \mathrm{d} t^{\prime}\right\}, \quad k=\frac{F t}{\hbar},
\end{gathered}
$$

(the hat over the exponent denotes time ordering). Substituting Eq. (18) into Eq. (17) the transition matrix elements are found as the Fourier image

$$
V_{\alpha, \beta}^{l, l^{\prime}}=2 \pi l \delta_{\alpha, \beta} \delta_{l, l^{\prime}}+\int_{-1 / 2}^{1 / 2} \mathrm{~d} k e^{i 2 \pi\left(l-l^{\prime}\right) k} Y_{\alpha, \beta}(k)
$$

of the periodic function

$$
Y_{\alpha, \beta}(k)=\frac{1}{F}\left\langle\phi_{\alpha}^{k}\left|\frac{(\hat{p}+k)^{2}}{2}+\cos x\right| \phi_{\beta}^{k}\right\rangle .
$$


A few remarks will be helpful concerning the numerical method of calculating the transition matrix elements (20). The numerical routine is organized as follows. First we find the right and left eigenvectors of the matrix $B$ (see Eq. (36) in Ref. [1])

$$
B \mathbf{c}_{\alpha}^{0}=\exp \left(-i \mathcal{E}_{\alpha} T_{B} / \hbar\right) \mathbf{c}_{\alpha}^{0}, \quad \mathbf{c}_{\alpha}^{0} B=\exp \left(-i \mathcal{E}_{\alpha} T_{B} / \hbar\right) \mathbf{c}_{\alpha}^{0} .
$$

Then the vectors $\mathbf{c}_{\alpha}^{0}$ are normalized as $\mathbf{c}_{\alpha}^{0} \rightarrow \mathbf{c}_{\alpha}^{0} /\left\langle\mathbf{c}_{\alpha}^{0} \mid \mathbf{c}_{\alpha}^{0}\right\rangle$. This procedure is somewhat delicate because the normalization constant $\left\langle\mathbf{c}_{\alpha}^{0} \mid \mathbf{c}_{\alpha}^{0}\right\rangle$ exponentially decreases when the dimension $N$ of the matrix $B$ is increased. On the other hand, the accuracy of the eigenvalues $\mathcal{E}_{\alpha}$ increases when $N \rightarrow \infty$. Thus one should keep a compromise and take the numerical parameter $N$ such as to get good accuracy for $\mathcal{E}_{\alpha}$ but not to loose the accuracy for the normalized eigenvectors $\mathbf{c}_{\alpha}^{0}$.

After the vectors $\mathbf{c}_{\alpha}^{0}$ are made orthogonal, $\left\langle\mathbf{c}_{\alpha}^{0} \mid \mathbf{c}_{\beta}^{0}\right\rangle=$ $\delta_{\alpha, \beta}$, they are evolved according to Eq. (18) (more precisely, according to its matrix counterpart) and the periodic function (21) is computed. Finally, the transition matrix elements (20) are found by a discrete Fourier transformation with typical step over $\Delta k=2^{-11}$.

\section{Transition matrix elements: Numerical example}

In this section we calculate the transition matrix elements between the $l$-th ground Wannier-Stark state and $l^{\prime}$-th first excited Wannier-Stark state. In our numerical example we fix the static field to the moderate value $F=0.04$ and change the value of the scaled Planck constant in the interval $1 \leq \hbar \leq 2.5$. The value of the scaled Planck constant [entering the momentum operator in the Hamiltonian (1)] obviously defines the positions and the number of stable resonances supported by each of the potential wells. In the experiment with cold atoms $\hbar$ is inverse proportional to the amplitude of the optical potential and can be varied by changing the intensity of the laser $[2,5]$.

We begin with small values of $\hbar$. For $\hbar \leq 1$ both Wannier-Stark states are essentially localized within a single potential well and can be approximated by the eigenfunctions of a harmonic oscillator. Then only "vertical" transitions $l=l^{\prime}$ (see Fig. 1) between the ground and first excited Wannier ladders are possible. By increasing $\hbar$ we move the Wannier levels towards the top of the potential barrier (for $\hbar>1.6$ the upper Wannier level is already above the potential barrier). Then the localization length of the Wannier states grows and more than one matrix element may differ from zero. Simultaneously with the increase of the localization length the Wannier states loose their stability. As a consequence the matrix elements $V_{\alpha, \beta}^{l, l^{\prime}}$ become complex and $V_{1,0}^{l^{\prime}, l} \neq\left(V_{0,1}^{l, l^{\prime}}\right)^{*}[9]$.

Figure 3 shows the absolute values of the coefficients (8) for $\hbar=1,1.5,2$, and 2.5. We recall that, according to the results of Sec. 2, these coefficients define the relative intensities of the lines in the total decay rate $\boldsymbol{\Gamma}(\omega)$. For the chosen values of the scaled Plank constant the width of the ground Wannier resonances are $\Gamma_{0}=2.31 \cdot 10^{-15}$, $1.06 \cdot 10^{-9}, 3.22 \cdot 10^{-6}$, and $6.08 \cdot 10^{-5}$, respectively. The upper resonances have the widths $\Gamma_{1}=1.90 \cdot 10^{-5}, 1.35$. $10^{-2}, 5.24 \cdot 10^{-2}$, and $1.14 \cdot 10^{-1}$. (These widths should be compared with the Bloch energy $\hbar \omega_{B}=2 \pi F \approx 0.25$. Since $\Gamma_{1} / \hbar \omega_{B}<1$, the resonances are well separated.) As expected, the number of lines is seen to increase with $\hbar$. A new result is the asymmetry of the coefficients with respect to $L$. It appears that the transitions "down the ladder" are enhanced in comparison with the transitions "up the ladder". At the same time, for the exponentially weak far transitions $(|L| \gg 1)$ the situation is reversed [see Fig. 4(a)].

Along with the transitions between the Wannier states belonging to different ladders the periodic driving causes transitions within one ladder. The right panel (b) in Fig. 4 shows the absolute value of the transition matrix elements $V_{0,0}(L)=V_{0,0}^{l, l^{\prime}}$ as the functions of the distance $L=l-l^{\prime}$ for $\hbar=2$. It is seen that these "horizontal" transition matrix elements are symmetric with respect to $L$ and decrease exponentially for large $|L|$ :

$$
\left|V_{\alpha, \alpha}(L)\right| \sim \exp \left(-|L| / \delta_{\alpha}\right) .
$$

The effect of the "horizontal" transitions becomes very important when the driving frequency $\omega$ coincides with the Bloch frequency $\omega_{B}$ (more generally, for $\omega / \omega_{B}=r / q$, where $r$ and $q$ are coprime integers). Then the quasienergy states are delocalized functions and the quasienergy spectrum has a band structure. The formation of the quasienergy band was studied in Ref. [7] by using a first order perturbation theory.

\section{Lineshapes of the decay spectra}

Knowing the coefficients (8) we can find the the decay spectra $\boldsymbol{\Gamma}(\omega)$. In fact, according to Eq. (12) the induced decay rate $\boldsymbol{\Gamma}(\omega)$ is given by the imaginary part of the most stable quasienergy. Since we consider the case $F_{\omega}\left|V_{0,1}(L)\right| \ll$ $\Gamma_{1}$, we can apply a perturbation approach to calculate the quasienergies. In zeroth order the most stable quasienergy coincides with the energy of the ground Wannier states taken modulo $\hbar \omega$. The first order gives correction to the real part of the quasienergy and correction to the imaginary part appears only in second order. Using the rotating wave approximation this correction is

$$
\boldsymbol{\Gamma}(\omega)=\operatorname{Im}\left(\sum_{L} \frac{F_{\omega}^{2} V_{0,1}^{2}(L)}{E_{1}-E_{0}+\hbar \omega_{B} L-\hbar \omega-i \Gamma_{1} / 2}\right) .
$$

Obviously, Eq. (24) generalizes Eq. (11) for the case of a many level system. The key difference, however, is that the coefficients $V_{0,1}^{2}(L)$ are complex now (see inset in Fig. 5). Thus the shape of the individual lines may differ from a Lorentz shape. This means that we meet a kind of Fano resonance, where the shape of the line is asymmetric. Using the notation from Fano theory [10], Eq. (24) can be 
presented in the form

$$
\Gamma(\omega)=F_{\omega}^{2} \sum_{L} A(L) \frac{q_{L}+\varepsilon_{L}}{1+\varepsilon_{L}^{2}} .
$$

In Eq. (25) $A(L)=2 \sin \phi(L)\left|V_{0,1}^{2}\right| / \Gamma_{1}, \varepsilon_{L}=2 \Delta_{0,1}(L) / \Gamma_{1}$, and the asymmetry part is given by $q_{L}=\cot \phi(L)$, where $\phi(L)$ is the phase of the coefficient $V_{0,1}^{2}(L)$. We note that the asymmetry of the lines was recently observed in an experiment with semiconductor superlattices [11].

The solid line in Fig. 5 shows the function (24) constructed for $\hbar=2$. This function exhibits all features of the decay spectra discussed above. Namely: the lines have the same width $\Gamma_{1}$ and are separated by the Bloch frequency $\omega_{B}$; the position of the lines is defined by the resonant condition $\omega=\left(E_{1}-E_{0}\right) / \hbar-\omega_{B} L(L$ is integer $)$ and their relative intensities are given by the absolute values of the coefficients $V_{0,1}^{2}(L)$ [compare with Fig. 3(c)]; the lines are asymmetric, which is especially well seen for the transitions $L=0$ and $L \geq 2$.

The dots in Fig. 5 show the results of an exact numerical calculation of the decay rate performed for some (rational) values of $\omega / \omega_{B}[5]$. A good correspondence indicates the validity of second order perturbation theory. The discrepancy in the region of small driving frequency is due to the rotating wave approximation, which obviously fails for $\omega \rightarrow 0$. As seen in Fig. 3 the envelope center for the transition coefficients moves to the left when $\hbar$ is increased. Thus the discrepancy discussed will be larger for $\hbar>2$. In principle, the region of small driving frequency requires a separate analysis.

In the rest of this section we discuss the effect of direct transitions to the second excited Wannier ladder. In second order perturbation theory we can take these transition into account by adding to the sum (24) the terms $F_{\omega}^{2} V_{0,2}^{2}(L) /\left(E_{2}-E_{0}+\hbar \omega_{B} L-\hbar \omega-i 0.5 \Gamma_{2}\right)$, where $V_{0,2}^{2}(L)=$ $V_{2,0}^{l, l^{\prime}} V_{0,2}^{l^{\prime}, l}$. For the considered case $\hbar=2, F=0.04$ the coefficients $V_{0,1}^{2}(L)$ and $V_{0,2}^{2}(L)$ are compared in the left column of Fig. 6 . It is seen that the main lines for $0 \leftrightarrow 2$ transitions [Fig. 6(c)] are ten times smaller than those for $0 \leftrightarrow 1$ [Fig. 6(a)]. Thus the effect of higher transitions can be neglected. We note, however, that this is not always the case. In the next section we consider a situation when the direct transitions to the second excited Wannier ladder cannot be ignored.

\section{Decay rate for $F_{\omega} \sim \omega^{2}$}

In Sec. 5 we have considered the case where the amplitude $F_{\omega}$ of the driving force is kept fixed when the frequency $\omega$ is varied. In this section we study the case $F_{\omega} \sim \omega^{2}$, which was actually realized in the experiment [3]. Indeed, in the experiment cited the authors measured the decay rate of the system

$$
\widehat{H}=\hat{p}^{2} / 2+\cos [x-\epsilon \cos (\omega t)]+F x .
$$

The Hamiltonian (26) is related to the Hamiltonian (1) by a Kramers-Henneberger transformation, where $\epsilon=F_{\omega} / \omega^{2}$.
Assuming $\epsilon \ll 1$ (which was the case in Ref. [3]) we approximate the time-periodic potential $\cos [x-\epsilon \cos (\omega t)]$ by $\cos x+\epsilon \cos (\omega t) \sin x$. Then the Hamiltonian (26) takes the form

$$
\widehat{H}=\widehat{H}_{0}+\epsilon \cos (\omega t) \sin x .
$$

Obviously, the decay rate of the system (27) is given by Eq. (24) where instead of the coefficients (8) one substitutes the coefficients

$$
W_{0,1}^{2}(L)=W_{0,1}^{l, l^{\prime}} W_{1,0}^{l^{\prime}, l}, \quad W_{\alpha, \beta}^{l, l^{\prime}}=\left\langle\Psi_{\alpha}^{l}|\sin x| \Psi_{\beta}^{l^{\prime}}\right\rangle .
$$

Using the operator identity

$$
\frac{1}{\hbar^{2}}\left[\widehat{H}_{0},\left[\widehat{H}_{0}, x\right]\right]=\sin x+F .
$$

we obtain the following relation between the coefficients (8) and (28)

$$
W_{0,1}^{2}(L)=\omega_{0,1}^{4}(L) V_{0,1}^{2}(L),
$$

where $\hbar \omega_{0,1}(L)=\mathcal{E}_{1}^{l^{\prime}}-\mathcal{E}_{0}^{l}$ is the energy difference between the considered Wannier-Stark levels. Thus, all we have to do is to multiply each term in sum (24) by a factor $\omega_{0,1}^{4}(L)$. The rescaling procedure (30) suppresses the transition "down the ladder" $(L<0)$ and enhances the transition "up the ladder" $(L>0)$.

The coefficients $W_{0,1}^{2}(L)$ and $W_{0,2}^{2}(L)$ are shown in the right column of Fig. 6 . Unlike the case $F_{\omega}=$ constant (left column in Fig. 6), in the presently considered case $\epsilon=$ constant the direct transitions to the second excited Wannier ladder can not be ignored. The analysis of the function $\boldsymbol{\Gamma}(\omega)$ confirms this conclusion. The solid and dashed lines in Fig.7 show the decay rates $\boldsymbol{\Gamma}(\omega)$ calculated with and without taking into account the higher transitions. It is seen that the solid line reproduces the exact numerical result (dots) much better than the dashed line.

\section{Conclusion}

We have applied a nonhermitian resonant perturbation theory to calculate the induced decay rate $\boldsymbol{\Gamma}(\omega)$ for a driven Wannier-Stark system (1)-(2). According to this theory the relative intensities of the peaks of $\boldsymbol{\Gamma}(\omega)$ are given by the the product of the absolute values of the "up" and "down" transition matrix elements, which can be calculated by a method developed in the present paper. We would like to stress that the complex phase of this product (which is zero in a hermitian approach) also has a physical meaning and defines the shape of individual lines of $\boldsymbol{\Gamma}(\omega)$. The effect of the phases becomes even more important, when (along with the transition $0 \leftrightarrow 1$ ) transitions to higher excited states are induced. In this case the function $\boldsymbol{\Gamma}(\omega)$ can show an interference pattern.

To avoid any misunderstanding it should be noted that in this present paper we actually ignored the "horizontal" transitions between the Wannier states belonging to 
the same ladder. This approximation is valid provided the commensurability condition

$$
\frac{\omega}{\omega_{B}}=\frac{r}{q}, \quad r, q=\text { integer },
$$

is avoided. In this sense the presented results are complementary to to the results of Refs. [5,7], where the commensurate case was studied in detail.

\section{References}

[*] Also at L. V. Kirensky Institute of Physics, 660036 Krasnoyarsk, Russia.

1. M. Glück, A. R. Kolovsky, and H. J. Korsch, "Resonant tunneling of the Wannier-Stark states", this issue.

2. M. Glück, A. R. Kolovsky, H. J. Korsch, Phys. Rev. Lett. 82, 1534 (1999).

3. S. R. Wilkinson, C. F. Bharucha, K. W. Madison, Qian $\mathrm{Niu}$, and M. G. Raizen, Phys. Rev. Lett. 76, 4512 (1996); see also K. W. Madison, M. C. Fisher, and M. G. Raizen, Phys. Rev. A 60, R1767 (1999), for recent progress in the experiment.

4. For a review, see E. E. Mendez and G. Bastard, Phys. Today 46, No. 6, (1993) 34; F. Rossi, Semicond. Sci. Technol. 13, 147 (1998); K. Leo, Semicond. Sci. Technol. 13, 249 (1998).

5. M. Glück, M. Hankel, A. R. Kolovsky, and H. J. Korsch, submitted to PRL.

6. M. Glück, A. R. Kolovsky, H. J. Korsch, and N. Moiseyev, EPJ D4, 239 (1998).

7. M. Glück, A. R. Kolovsky, and H. J. Korsch, Phys. Lett. A 258, 383 (1999).

8. In the paper [5] the same result was concluded on the basis of the Fermi golden rule.

9. Strictly speaking the matrix elements are real only in the limit $\hbar \rightarrow 0$.

10. U. Fano, Phys. Rev. 124, 1866 (1961).

11. C. P. Holfeld, F. Löser, M. Sudzius, K. Leo, D. M. Whittaker, and K. Köhler, Phys. Rev. Lett. 81, 874 (1998).

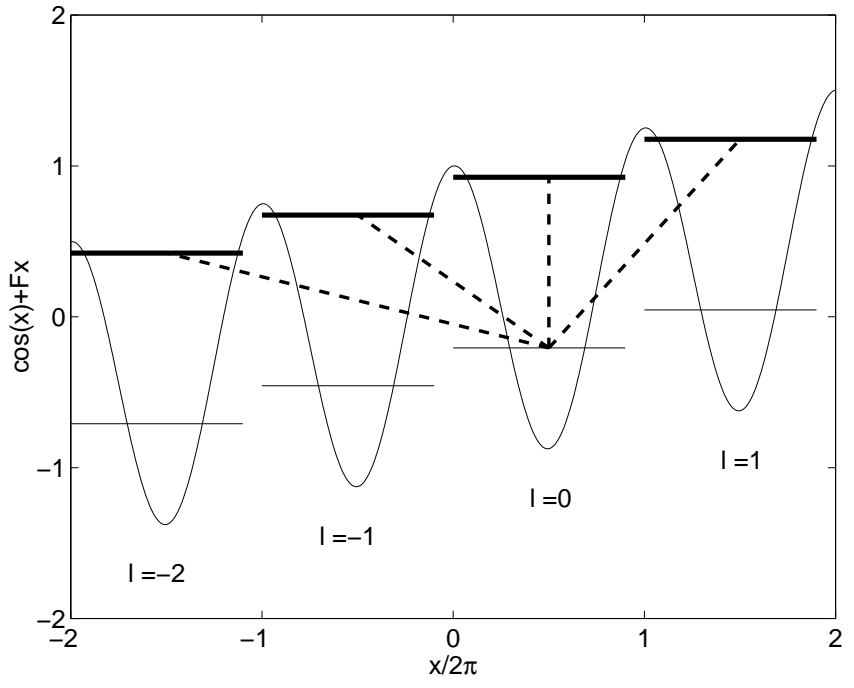

Fig. 1. Scheme of transitions in the system (1) induced by the periodic driving. The positions of the ground $\left(E_{0}^{l}\right)$ and the first excited $\left(E_{1}^{l}\right)$ Wannier levels are shown for $F=0.04$ and $\hbar=1.5$.
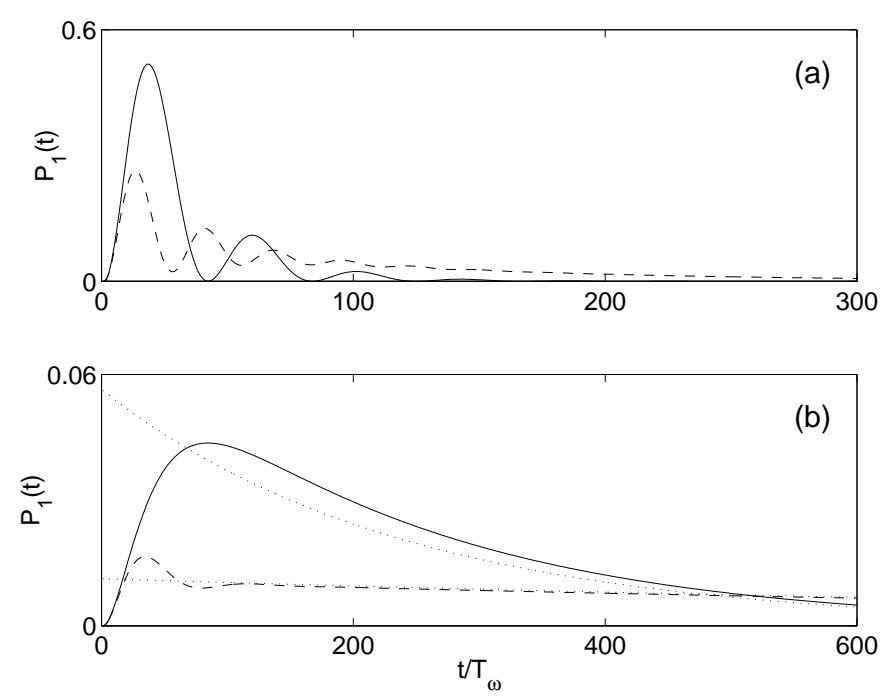

Fig. 2. Dynamics of the population of the upper level in the two-level system (4) in the case of decaying Rabi oscillations (a) and in the overdamped case (b). The parameters are chosen to correspond to the "vertical" transition $(L=0)$ in the system (1) for $F=0.04, \hbar=1.5, F_{\omega}=0.04$ (a), and $F_{\omega}=0.004$ (b). The solid lines are the case of zero detuning, the dashed lines correspond to $\Delta_{0,1}(0)=F_{\omega}\left|V_{0,1}\right|$ (a) and $\Delta_{0,1}(0)=\Gamma_{1}(\mathrm{~b})$, the dotted line shows the asymptotic solution (10)- (11). 

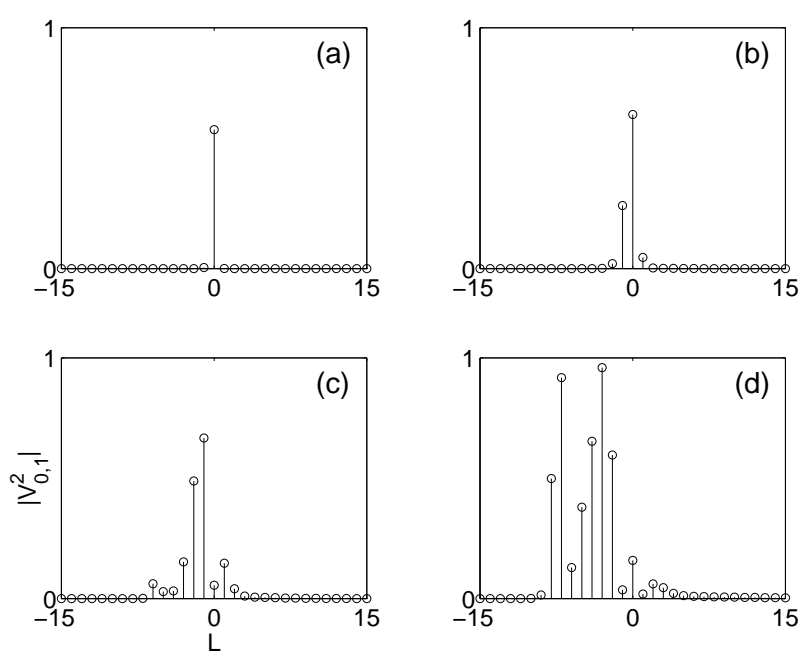

Fig. 3. The absolute values $\left|V_{0,1}^{2}(L)\right|$ of the expansion coefficients (8) for $F=0.04$ and $\hbar=1$ (a), 1.5 (b), 2 (c), and 2.5 (d). These quantities define the relative intensities of the lines of the function $\Gamma(\omega)$.
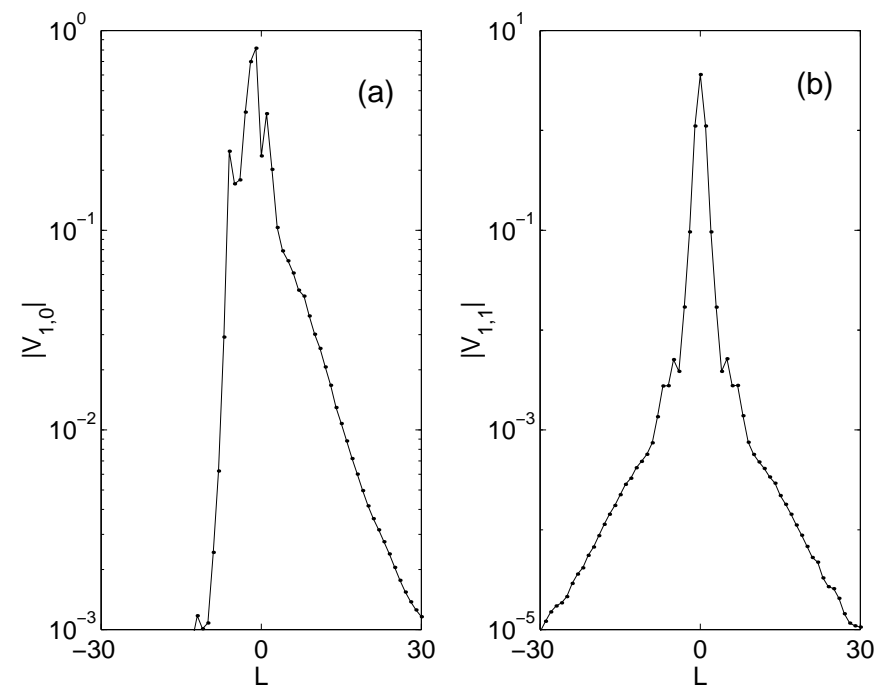

Fig. 4. The absolute values of the matrix elements $V_{0,1}^{l, l^{\prime}}$ (a) and $V_{0,0}^{l, l^{\prime}}$ (b) as the functions of $L=l-l^{\prime}$ for $\hbar=2$ in logarithmic scale.

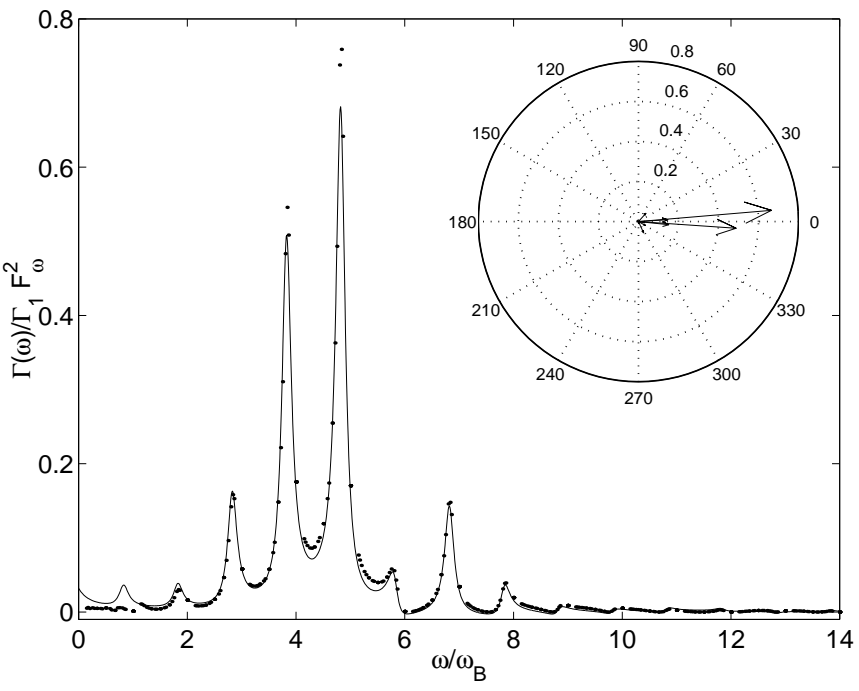

Fig. 5. Comparison of the second order approximation (24) (solid line) with the exact numerical calculation of the system decay rate (dots) for $\hbar=2, F_{\omega}=0.02$. The inset shows the expansion coefficients (8) in the angle plane.
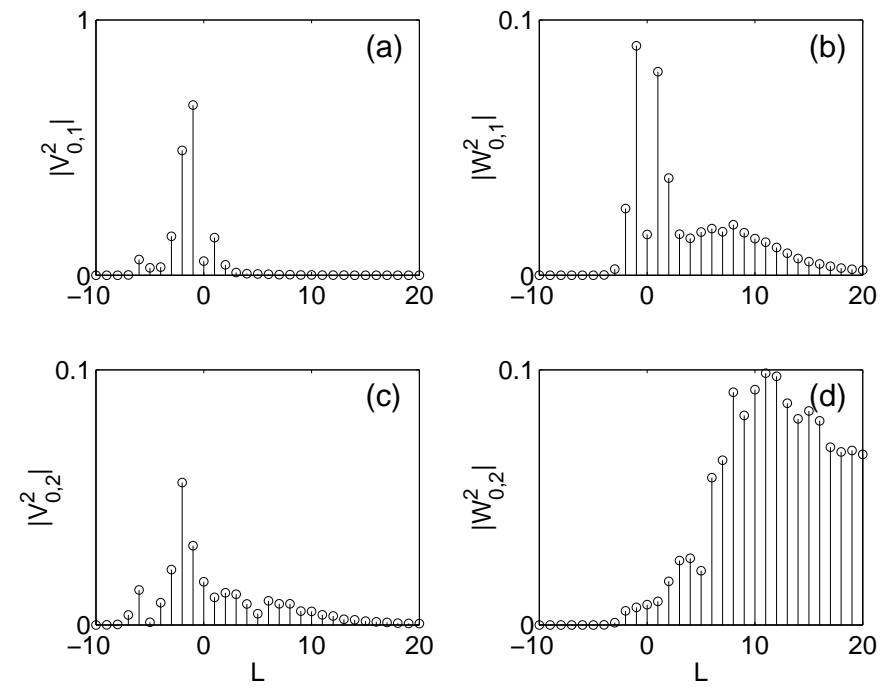

Fig. 6. The absolute values of the coefficients $V_{0,1}^{2}(L)$ (a), $W_{0,1}^{2}(L)(\mathrm{b}), V_{0,2}^{2}(L)(\mathrm{c})$, and $W_{0,2}^{2}(L)(\mathrm{d})$. 


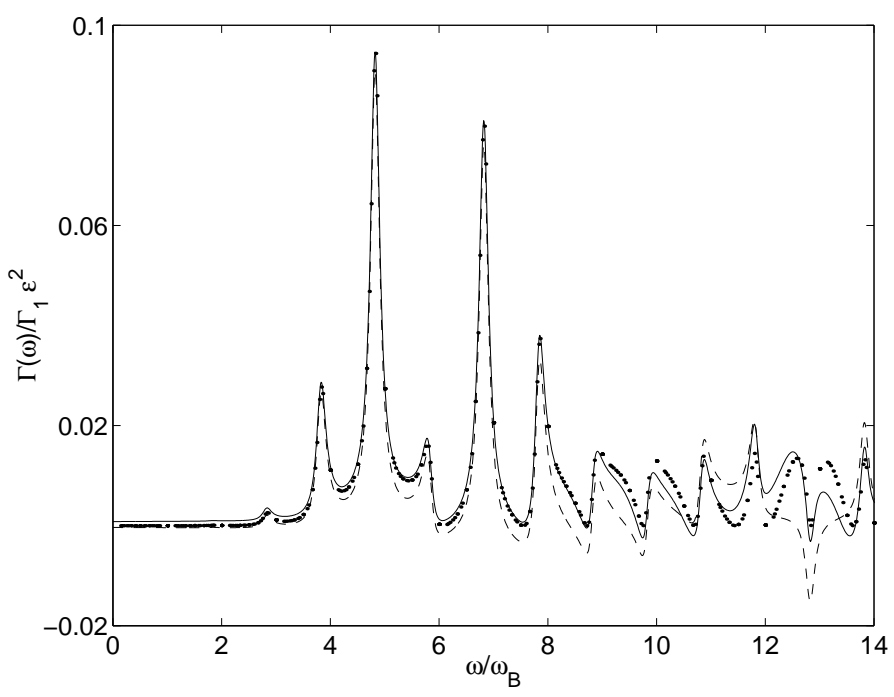

Fig. 7. The decay rate of the system (30) for $\hbar=2, F=0.04$, and $\epsilon=0.02$. Dots are the exact result, the solid and dashed lines are calculated by using second order perturbation theory with and without taking into account the direct transitions to the second excited Wannier ladder. 